\title{
Comparação dos graus de fibrose hepática na hepatite C crônica (HCC) medidos por métodos de elastrografia e de sorologia: ARFI e FibroScan vs APRI e FIB4
}

\section{Comparison of hepatitis C chronic (HCC) degrees of fibrosis hepatic measured by methods of elastrography and sorologycs: ARFI and FibroScan vs APRI and FIB4}

\author{
Flávia Siqueira Furtado Melloํ․ José Milton de Castro Limaํ. Elodie Bomfim Hyppolito². Rodrigo Vieira \\ Costa Lima². Flávio Esmeraldo Rolim ${ }^{2}$ Cibele Silveira Pinho ${ }^{1}$. Jesus Irajacy Fernandes da Costa ${ }^{1}$. \\ 1 Universidade Federal do Ceará (UFC), Fortaleza, Ceará, Brasil. 2 Hospital Universitário Walter Cantídio (HUWC), Fortaleza, \\ Ceará, Brasil.
}

\section{RESUMO}

Para tratar a hepatite C crônica (HCC) deve-se conhecer o grau de fibrose hepática, cuja melhor determinação obtém-se por biopsia hepática. Contudo, métodos não-invasivos elastográficos e sorológicos têm sido preferidos. Objetivos: Comparar a concordância entre dois métodos elastográficos e dois sorológicos. Metodologia: Entre janeiro/2014-dezembro/2016 selecionamos portadores de HCC estadiados por FibroScan ou ARFI. Seus graus fibróticos foram agrupados (F0/F1; F2/F3/F4) e comparados aos graus fibróticos calculados laboratorialmente por APRI e FIB4 e agrupados (F0/F1; F2/F3/F4). Comparamos, FibroScan com APRI, FibroScan com FIB-4, ARFI com APRI, ARFI com FIB-4 e APRI com FIB4. Análises: concordância Kappa. Resultados: 73 pacientes (54 masculinos); idade média: 58 anos (32-81), estadeados por FibroScan (31) e ARFI (42). Para comparações, calculou-se APRI e FIB4. Entre FibroScan e APRI ou FIB4 há melhor concordância em graus fibróticos acentuados (grupo F2/F3/ F4). APRI e FIB4 subestimaram a fibrose em relação ao FibroScan. ARFI comparado a APRI e FIB4 mostrou pobre concordância. Concordância entre ARFI e APRI é melhor em graus fibróticos baixos (grupo F0/F1) e é ausente em graus elevados (grupo F2/F3/ F4). O FIB4 superestima a fibrose em relação ao ARFI. Conclusão: A concordância entre métodos sorológicos e elastográficos varia desde a ausente de concordância até concordância moderada.

Palavras-chave: Elastografia. Hepatite C crônica. Fibrose hepática.

\begin{abstract}
For treating chronic hepatitis $\mathrm{C}(\mathrm{CHC})$ we should know the hepatic fibrosis degree, which is better evaluated through hepatic biopsy. However, non-invasive elastography and serological methods have being preferred. Aims: Compare the concordance between two elastography and two serologicals methods. Methodology: From January/2014-December/2016 CHC patients with elastography (FibroScan and ARFI) were enrolled. Their fibrotic degrees were grouped (F0/F1; F2/F3/F4) and compared with fibrotic degrees calculated by APRI and FIB4, grouped (F0/F1; F2/F3/F4). Comparisons: FibroScan with APRI, FibroScan with FIB-4, ARFI with APRI, ARFI with FIB-4 e APRI with FIB4. Analysis: Kappa concordance. Results: 73 patients (54 male); mean age of 58 (32 - 81) years-old, had elastography (31 FibroScan and 42 ARFI). For comparisons APRI and FIB4 were calculated. Comparisons between FibroScan and APRI or FIB4 showed better concordance in high degrees of fibrosis (group F2/F3/F4). APRI and FIB4 underestimated the fibrosis in relation to FibroScan. ARFI compared to APRI and FIB4 showed poor concordance. Concordance between ARFI and APRI is better in the low degrees (group F0/F1) and is absent in high degrees of fibrosis (group F2/F3/F4). FIB4 overestimated the fibrosis in relation to ARFI. Conclusion: The concordance of serologicals and elastography methods vary from an absence of concordance to a moderate concordance.
\end{abstract}

Keywords: Elastography. Chronic hepatitis C. Liver fibrosis.

Autor correspondente: Flávia Siqueira Furtado Mello, Rua Manuel Jacaré, 190, Mucuripe, Fortaleza, Ceará. CEP: 60175-110. Telefone: +55 85 99919044. E-mail: flaviaf@me.com

Conflito de interesses: Não há qualquer conflito de interesses por parte de qualquer um dos autores.

Recebido em: 19 Out 2018; Revisado em: 01 Ago 2019; Aceito em: 02 Ago 2019. 


\section{INTRODUÇÃO}

A infecção pelo vírus da hepatite C (VHC) afeta cerca de 170 milhões de pessoas, aproximadamente $3 \%$ da população mundial. Estima-se que $54 \%$ a $86 \%$ dos pacientes infectados evoluam para a forma crônica, a qual predispõe a complicações, como cirrose hepática, falência hepática e hepatocarcinoma. ${ }^{1}$

Calcula-se que a prevalência de pessoas soro-reagentes (anti-VHC positivo) seja de aproximadamente $0,7 \%$, o que corresponde a 1.032.000 somente no Brasil. Dessas, estima-se que 657.000 sejam virêmicas, e que necessitem de tratamento. Esclarecemos que essa estimativa de prevalência de $0,7 \%$ é referente à população geral entre 15 e 69 anos, avaliada até $2016 .{ }^{1}$

Em 2018, foi publicado em Brasília o novo Protocolo de Tratamento da Hepatite C, que mudou os critérios de inclusão para terapia com medicamentos antivirais de ação direta (DAAs). ${ }^{1}$ Em 2015, o tratamento era previsto para pacientes coinfectados com HIV, pacientes com manifestações extrahepáticas, pacientes transplantados de órgãos sólidos, pacientes com cirrose hepática, pacientes com biópsia hepática realizada há pelo menos 2 anos e com grau de fibrose F2 e aqueles com graus de fibrose F3 e/ou F4 determinados por biópsias hepáticas, métodos de elastografia hepática (FibroScan ou ARFI) ou métodos de coeficientes laboratoriais APRI e FIB4. ${ }^{2}$

Atualmente, pelo protocolo de 2018, o tratamento é previsto para todos os pacientes portadores de hepatocarcinoma (HCC) independentemente do grau de fibrose hepática, ${ }^{1}$ porém, sua aferição ainda é importante para determinar a duração do tratamento e, em alguns casos, a escolha dos medicamentos a serem utilizados. Além disso, o grau de fibrose tem grande valor prognóstico. ${ }^{1}$

É importante determinar a existência de doença avançada (Cirrose Hepática), a qual se caracteriza pela presença de circulação colateral, fígado irregular e reduzido de tamanho, esplenomegalia, aumento do calibre da veia porta, redução do fluxo portal, ascite e varizes esofágicas. ${ }^{1,2}$

Recomenda-se que o estadiamento da fibrose hepática seja realizado para todos os portadores de hepatite $\mathrm{C}$, coinfectados ou não pelo HIV, de modo a caracterizar ausência ou presença de doença avançada. ${ }^{1,2}$ Este estadiamento pode ser realizado por qualquer um dos seguintes métodos: biópsia hepática, FibroScan, ARFI, ressonância magnética, APRI, FIB4, APRI modificado, Fibrotest, Fornix Index, dentre outros. ${ }^{3,4,5}$

No Brasil, são aceitos os métodos disponíveis no SUS: biópsia hepática, FibroScan, ARFI, APRI e FIB4.${ }^{1}$ A biópsia hepática é considerada o padrão ouro, ${ }^{6}$ porém, é um método invasivo que envolve risco de complicações, como sangramento, fístula biliar, perfuração, infecção, dor local e raros óbitos. Necessita a obtenção de amostra de tecido hepático com pelo menos 10 espaços porta para um resultado conclusivo ${ }^{6}$ e que tem possibilidade de divergência de interpretação de resultados, mesmo entre patologistas experientes. ${ }^{5}$ Por essas limitações da biópsia hepática é que se buscam outros métodos de estadiamento da fibrose hepática. O teste ideal deve ter boa acurácia, baixo potencial de complicações, ser replicável, ter baixo custo e ser acessível até mesmo em países subdesenvolvidos. ${ }^{3}$

Dentre os métodos não invasivos têm-se aqueles por imagem (FibroScan, ARFI, Elastografia em Tempo Real, Ressonância Magnética) ${ }^{3,4,5}$ e os métodos laboratoriais (APRI, FIB-4). ${ }^{3,4,5}$ Dos métodos de imagem, a elastografia apresenta como principais vantagens a possibilidade de avaliação de uma área bem maior do parênquima hepático do que a avaliada em limitado fragmento de biópsia hepática, além disso oferece resultados que reproduzam a real situação do parênquima. ${ }^{3,4}$ Suas principais limitações são o caráter de operador dependência, o custo elevado e a potencial interferência em seus resultados nas situações que aumentam a rigidez hepática, independentemente de fibrose, como: elevada atividade necroinflamatória (alanina aminotransferase $($ ALT) $>5 \mathrm{x}$ o limite superior da normalidade (LSN)), excesso de ingestão de bebidas alcoólicas, colestase extra-hepática, congestão hepática, alto grau de esteatose/obesidade, ausência de jejum adequado (pelo menos de quatro horas), doenças de depósito e infiltração celular anômala no parênquima hepático. Ademais, o desempenho da elastografia não é bom em obesos (IMC $>30 \mathrm{~kg} / \mathrm{m}^{2}$ ), nos quais há falhas de interpretação em aproximadamente 20\% dos $\operatorname{casos}^{3,7}$ e também não se presta a cirróticos e a portadores de outras alterações de parênquima ou função hepática, como esquistossomose e leishmaniose. ${ }^{7}$ Os valores relativos aos diferentes estágios da fibrose hepática, obtidos por elastografia, dependem do método e do equipamento utilizado. Os métodos elastográficos que utilizam a ultrassonografia (FibroScan e ARFI) são os mais recomendados internacionalmente para a avaliação do estadiamento de fibrose hepática. ${ }^{5} \mathrm{O}$ FibroScan é o mais difundido e validado no mundo. ${ }^{4}$ A mensuração da fibrose hepática é baseada em ondas vibratórias emitidas de um transdutor. A velocidade com que essas ondas atravessam o tecido e retornam ao transdutor é mensurada e usada para o cálculo do grau de fibrose. Quanto maior o grau de fibrose, mais rápida a propagação da onda. ${ }^{4}$

O FibroScan possui a desvantagem de perder acurácia em obesos, em pacientes com insuficiência cardíaca classe III ou IV, esteatose importante, inflamação hepática considerável (AST maior que $100 \mathrm{U} / \mathrm{L}$ ), colestase elevada, níveis altos de bilirrubina e em pacientes com ascite. O método é capaz de identificar presença de fibrose significante (maior ou igual a F2) com valor de corte de 7,8 $\mathrm{kPa}$, com sensibilidade de $88 \%$ e especificidade de $100 \%{ }^{5}$

O ARFI (Acoustic Radiation Force Imaging) é outro método eletrográfico não invasivo, economicamente mais viável que a biópsia hepática, e que possui como vantagem o fato de ser integrado a um ultrassom convencional, o que permite ao médico, além de obter o grau de fibrose, efetuar a avaliação ultrassonográfica e do doppler dos vasos hepáticos. Ele utiliza um software, que através do transdutor convencional de ultrassom, com 3,5 MHz, sem necessidade de compressão, emite ondas 10.000 vezes mais rápidas em relação às ondas convencionais. A propagação das ondas é seguida pela 
detecção de pulsos, cuja mensuração determina a velocidade de propagação da onda, que é diretamente relacionada ao grau de rigidez hepática. O grau de fibrose é classificado em F0, F1, F2, F3 e F4. ${ }^{6,7}$ As doenças que superestimam a elastografia ARFI são congestão hepática, colestase hepática, hepatite aguda e IMC acima de $40 .{ }^{6,7}$ Inúmeros estudos, que comparam o ARFI ao FibroScan, método este já bastante validado para mensuração da fibrose hepática em pacientes com HCC, mostram que eles são comparáveis em sensibilidade e especificidade ${ }^{8}$ e que ambos são comparáveis à biópsia hepática. ${ }^{4}$

Dentre os métodos laboratoriais o APRI (AST Platelet Ratio Index) é bastante atraente, pois, além de utilizar uma fórmula de fácil aplicação com índices laboratoriais simples e muito disponíveis, tem demonstrado ser um teste de elevada acurácia como preditor da fibrose hepática nos portadores de HCC. ${ }^{9,10}$ Estudos mostram que o APRI foi capaz de prever fibrose significativa (F2 ou superior) com sensibilidade de $54 \%$ e especificidade de $80 \% .{ }^{10}$ Utiliza apenas marcadores bioquímicos (AST U/Le Plaquetas 10/L). Como desvantagem, assinala-se o fato de os níveis de AST e de plaquetas serem influenciados por viroses (infecção por HIV, Vírus da Hepatite B e outros vírus), níveis de gordura hepática, agressões imunes, ação de medicamentos, etc. ${ }^{11}$

O FIB4 (fibrosis 4) é outro método laboratorial, que também utiliza fórmula de fácil aplicação baseada em índices laboratoriais simples, além da idade do paciente. Revisões já demonstraram que o FIB4 permite a detecção de fibrose F3 e F4 com um valor preditivo positivo de $82,1 \%$, para valores maiores que 3,25 , e para valores menores que 1,45 , valor preditivo negativo de $94,7 \%$ para excluir fibrose severa. ${ }^{8}$ Os valores correspondentes ao grau de fibrose para os métodos sorológicos são demonstrados no Quadro 1. ${ }^{1}$

Nesse estudo avaliamos dois métodos de elastografia (por imagem), FibroScan e ARFI, e dois métodos laboratoriais, FIB4 e APRI.

Quadro 1. Caracterização de fibrose hepática conforme valores de APRI e FIB4. ${ }^{1}$

\begin{tabular}{|c|c|c|c|}
\hline \multicolumn{4}{|c|}{ APRI (avaliação de fibrose hepática avançada) } \\
\hline Resultado & $<0,5$ & $0,5-1,49$ & $\geq 1,5$ \\
\hline Interpretação & $\begin{array}{l}\text { Baixa probabilidade de } \\
\text { fibrose hepática F2, F3 } \\
\text { ou F4 }\end{array}$ & $\begin{array}{l}\text { Não é possível determinar } \\
\text { o estágio de fibrose } \\
\text { hepática }\end{array}$ & $\begin{array}{l}\text { Alta probabilidade de } \\
\text { fibrose hepática F3 ou F4 }\end{array}$ \\
\hline \multicolumn{4}{|c|}{ APRI (avaliação de cirrose hepática) } \\
\hline Resultado & $<1,0$ & $1,0-1,49$ & $\geq 2,0$ \\
\hline Interpretação & $\begin{array}{l}\text { Baixa probabilidade de } \\
\text { cirrose (F4) }\end{array}$ & $\begin{array}{l}\text { Não é possível determinar } \\
\text { o estágio de fibrose } \\
\text { hepática }\end{array}$ & $\begin{array}{l}\text { Alta probabilidade de } \\
\text { cirrose (F4) }\end{array}$ \\
\hline \multicolumn{4}{|c|}{ FIB-4 (avaliação de fibrose hepática avançada ou cirrose) } \\
\hline Resultado & $<1,45$ & $1,45-3,24$ & $\geq 3,25$ \\
\hline Interpretação & $\begin{array}{l}\text { Baixa probabilidade de F2, } \\
\text { F3 ou F4 }\end{array}$ & $\begin{array}{l}\text { Não é possível determinar } \\
\text { o estágio de fibrose } \\
\text { hepática }\end{array}$ & $\begin{array}{l}\text { Alta probabilidade de F3 } \\
\text { ou F4 }\end{array}$ \\
\hline
\end{tabular}

\section{METODOLOGIA}

Estudo comparativo, retrospectivo, transversal, no qual foram analisados 554 pacientes portadores de HCC, provenientes dos ambulatórios de Gastrenterologia do Hospital Universitário Walter Cantídio (HUWC), Pré-transplante Hepático do HUWC, Pós-transplante Hepático do HUWC e Ambulatório de Hepatites do Hospital São José (HSJ), acompanhados de janeiro 2014 a dezembro de 2016. Todos os pacientes foram informados da realização do estudo. O trabalho foi submetido e aprovado pelos comitês de éticas das referidas instituições. Os pacientes do estudo eram aqueles elegíveis para realizar o tratamento da Hepatite C com os novos DAAs (Sofosbovir, Daclastavir e Simeprevir). Setenta e três pacientes, sendo 54 do sexo masculino, 19 do sexo feminino, com idades variando entre 32 e 81 anos, com média de idade de 58 anos, possuíam estadiamento de grau de fibrose por ARFI ou FibroScan foram selecionados para análise no presente estudo.
Foram realizados 42 exames de ARFI por apenas um examinador experiente (mais de 100 exames realizados previamente) e 31 exames de FibroScan realizados por três examinadores diferentes, igualmente experientes. Foram analisados somente os exames que possuíam discriminação da data em que foram realizados e que precederam o início do tratamento antiviral. Para título de análise comparativa os resultados das elastografias foram divididos em 2 grupos F0/ F1 e F2/ F3/F4, assim como os resultados da mensuração de fibrose por métodos sorológicos.

Analisaram-se ainda os exames laboratoriais: transaminase glutamico oxalacetica (AST) U/L e ALT U/L e plaquetas, colhidos nos laboratórios do HUWC e do HSJ, antes do início do tratamento da Hepatite C. A partir desses exames, foram calculados o APRI e FIB4, segundo as fórmulas: APRI $=[(\mathrm{AST} \mathrm{U} / \mathrm{L} /$ valor superior de normalidade de AST U/L $) /$ Plaquetas $\left.10^{9} / \mathrm{L}\right]$ x 100 e FIB $4=($ idade $\times$ AST U/L) $/($ plaquetas 
$10^{9} / \mathrm{L}$ x $\sqrt{ }$ ALT U/L). O valor superior de normalidade de AST utilizado foi $38 \mathrm{U} / \mathrm{L}$ para homens e $32 \mathrm{U} / \mathrm{L}$ para mulheres. A interpretação dos valores de APRI e FIB4 foram realizados segundo o Quadro 1.

Foram critérios de exclusão: pacientes que possuíam mais de 2 anos de diferença entre a realização do método de elastografia e os exames laboratoriais, pacientes que não possuíam a data da realização do método de elastografia e pacientes que não possuíam data dos exames laboratoriais.

\section{RESULTADOS}

Os resultados dos métodos sorológicos foram analisados e divididos segundo F0, F1, F2, F3 e F4 (tabela 1), porém, a título de comparação com os métodos de imagem, divididos em F0/F1e F2/F3/F4. Os resultados foram analisados utilizando categoria Kappa, sendo realizadas as seguintes comparações: FibroScan + ARFI vs APRI (comparação na tabela 2 e resultados de concordância na tabela 3), FibroScan + ARFI vs FIB4 (comparação na tabela 4 e resultados de concordância na tabela 5); APRI vs FIB4 (comparação na tabela 6 e resultados de concordância na tabela 7); FibroScan vs APRI (comparação na tabela 8 e resultados de concordância na tabela 9); FibroScan $v s$ FIB4 (comparação na tabela 10 e resultados de concordância na tabela 11); ARFI vs APRI (comparação na tabela 12 e resultados de concordância na tabela 13); ARFI vs FIB4 (comparação na tabela 14 e resultados de concordância na tabela 15$)$.

Tabela 1. Interpretação dos resultados dos métodos sorológicos de mensuração de fibrose hepática.

\begin{tabular}{|l|l|l|}
\hline & APRI & FIB4 \\
\hline F0/F1 & $<0,5$ & $<1,45$ \\
\hline F2 & $0,5-1,5$ & $1,45-3,25$ \\
\hline F3/F4 & $>1,5$ & $>3,25$ \\
\hline
\end{tabular}

Tabela 2. Comparação FibroScan + ARFI vs APRI.

\begin{tabular}{|l|l|l|l|l|l|}
\hline \multicolumn{2}{|c|}{} & \multicolumn{5}{c|}{ APRI } \\
\cline { 3 - 7 } \multicolumn{2}{|c|}{} & F0/F1 & F2 & F3/F4 & TOTAL \\
\hline \multirow{4}{*}{ FibroScan +ARFI } & F0/F1 & 6 & 13 & 5 & 24 \\
\cline { 2 - 7 } & F2 & 0 & 5 & 3 & 8 \\
\cline { 2 - 7 } & F3/F4 & 3 & 24 & 15 & 42 \\
\cline { 2 - 7 } & TOTAL & 9 & 42 & 23 & 74 \\
\hline
\end{tabular}

Tabela 3. Resultado da Comparação do FibroScan + ARFI vs APRI, segundo categoria Kappa.

\begin{tabular}{|l|l|l|l|l|}
\hline & \multicolumn{1}{|c|}{ F0/F1 } & \multicolumn{1}{c|}{ F2 } & \multicolumn{1}{c|}{ F3/F4 } & \multicolumn{1}{c|}{ Geral } \\
\hline KAPPA DA CATEGORIA & 0.227 & 0.022 & 0.1 & 0.13 \\
\hline RESULTADOS & Fraca concordância & Pobre concordância & Pobre concordância & Pobre concordância \\
\hline
\end{tabular}

Tabela 4. Comparação FibroScan + ARFI vs FIB4.

\begin{tabular}{|l|l|l|l|l|l|}
\hline \multicolumn{2}{|c|}{} & \multicolumn{5}{c|}{ FIB-4 } \\
\cline { 3 - 7 } \multicolumn{2}{|c|}{} & F0/F1 & F2 & F3/F4 & TOTAL \\
\hline \multirow{4}{*}{ FibroScan + ARFI } & F0/F1 & 4 & 16 & 5 & 25 \\
\cline { 2 - 7 } & F2 & 0 & 6 & 2 & 8 \\
\cline { 2 - 7 } & F3/F4 & 3 & 22 & 15 & 40 \\
\cline { 2 - 6 } & TOTAL & 7 & 44 & 22 & 73 \\
\hline
\end{tabular}

Tabela 5. Resultado da comparação do FibroScan + ARFI vs FIB4, segundo categoria Kappa.

\begin{tabular}{|l|l|l|l|l|}
\hline & F0/F1 & F2 & F3/F4 & Geral \\
\hline KAPPA DA CATEGORIA & 0.118 & 0.056 & 0.155 & 0.107 \\
\hline RESULTADOS & Pobre concordância & Pobre concordância & Pobre concordância & Pobre concordância \\
\hline
\end{tabular}


Tabela 6. Comparação APRI vs FIB4.

\begin{tabular}{|l|l|l|l|l|l|}
\hline \multicolumn{2}{|c|}{} & \multicolumn{5}{c|}{ APRI } \\
\cline { 3 - 7 } \multicolumn{2}{c|}{} & F0/F1 & F2 & F3/F4 & TOTAL \\
\hline \multirow{3}{*}{ FIB-4 } & F0/F1 & 6 & 3 & 0 & 9 \\
\cline { 2 - 6 } & F2 & 4 & 36 & 4 & 44 \\
\cline { 2 - 6 } & F3/F4 & 0 & 3 & 18 & 21 \\
\cline { 2 - 6 } & TOTAL & 10 & 42 & 22 & 74 \\
\hline
\end{tabular}

Tabela 7. Resultado da comparação do APRI vs FIB4, segundo categoria Kappa.

\begin{tabular}{|l|l|l|l|l|}
\hline & F0/F1 & F2 & F3/F4 & GERAL \\
\hline KAPPA DA CATEGORIA & 0.577 & 0.612 & 0.771 & 0.663 \\
\hline RESULTADOS & $\begin{array}{l}\text { Concordância } \\
\text { moderada }\end{array}$ & $\begin{array}{l}\text { Concordância } \\
\text { importante }\end{array}$ & $\begin{array}{l}\text { Concordância } \\
\text { importante }\end{array}$ & $\begin{array}{l}\text { Concordância } \\
\text { importante }\end{array}$ \\
\hline
\end{tabular}

Tabela 8. Comparação FibroScan vs APRI.

\begin{tabular}{|l|l|l|l|l|l|}
\hline \multicolumn{2}{|c|}{} & \multicolumn{5}{c|}{ APRI } \\
\cline { 3 - 7 } \multicolumn{2}{|c|}{} & F0/F1 & F2 & F3/F4 & TOTAL \\
\hline \multirow{3}{*}{ FibroScan } & F0/F1 & 1 & 6 & 1 & 8 \\
\cline { 2 - 7 } & F2 & 0 & 3 & 1 & 4 \\
\cline { 2 - 7 } & F3/F4 & 0 & 11 & 8 & 19 \\
\cline { 2 - 6 } & TOTAL & 1 & 20 & 10 & 31 \\
\hline
\end{tabular}

Tabela 9. Resultado da comparação do FibroScan vs APRI, segundo categoria Kappa.

\begin{tabular}{|l|l|l|l|l|}
\hline & F0/F1 & F2 & F3/F4 & Geral \\
\hline KAPPA DA CATEGORIA & 0.175 & 0.045 & 0.224 & 0.138 \\
\hline RESULTADOS & Concordância pobre & Concordância pobre & Concordância fraca & Concordância pobre \\
\hline
\end{tabular}

Tabela 10. Comparação com FibroScan vs FIB4.

\begin{tabular}{|l|l|l|l|l|l|}
\hline \multicolumn{2}{|c|}{} & \multicolumn{5}{c|}{ FIB-4 } \\
\cline { 3 - 7 } \multicolumn{2}{c|}{} & F0/F1 & F2 & F3/F4 & TOTAL \\
\hline \multirow{3}{*}{ FibroScan } & F0/F1 & 1 & 6 & 1 & 8 \\
\cline { 2 - 7 } & F2 & 0 & 3 & 1 & 4 \\
\cline { 2 - 6 } & F3/F4 & 2 & 10 & 7 & 19 \\
\cline { 2 - 6 } & TOTAL & 3 & 19 & 9 & 31 \\
\hline
\end{tabular}

Tabela 11. Resultado da comparação do FibroScan vs FIB4, segundo categoria Kappa.

\begin{tabular}{|l|l|l|l|l|}
\hline & F0/F1 & F2 & F3/F4 & Geral \\
\hline KAPPA DA CATEGORIA & 0.048 & 0.061 & 0.175 & 0.101 \\
\hline RESULTADOS & Concordância pobre & Concordância pobre & Concordância pobre & Concordância pobre \\
\hline
\end{tabular}


Tabela 12. Comparação ARFI vs APRI.

\begin{tabular}{|l|l|l|l|l|l|}
\hline \multicolumn{2}{|c|}{} & \multicolumn{5}{c|}{ APRI } \\
\cline { 3 - 7 } \multicolumn{2}{c|}{} & F0/F1 & F2 & F3/F4 & TOTAL \\
\hline \multirow{4}{*}{ ARFI } & F0/F1 & 5 & 7 & 4 & 16 \\
\cline { 2 - 7 } & F2 & 0 & 2 & 2 & 4 \\
\cline { 2 - 7 } & F3/F4 & 3 & 13 & 6 & 22 \\
\cline { 2 - 6 } & TOTAL & 8 & 22 & 12 & 42 \\
\hline
\end{tabular}

Tabela 13. Resultado da comparação do ARFI vs APRI, segundo categoria Kappa.

\begin{tabular}{|l|l|l|l|l|}
\hline & F0/F1 & F2 & F3/F4 & Geral \\
\hline KAPPA DA CATEGORIA & 0.218 & -0.009 & -0.027 & 0.051 \\
\hline RESULTADOS & Concordância fraca & Não concordam & Não concordam & Concordância pobre \\
\hline
\end{tabular}

Tabela 14. Comparação ARFI vs FIB4.

\begin{tabular}{|l|l|l|l|l|l|}
\hline \multicolumn{2}{|c|}{} & \multicolumn{5}{c|}{ FIB-4 } \\
\cline { 3 - 7 } \multicolumn{2}{c|}{} & F0/F1 & F2 & F3/F4 & TOTAL \\
\hline \multirow{4}{*}{ ARFI } & F0/F1 & 3 & 9 & 4 & 16 \\
\cline { 2 - 7 } & F2 & 0 & 3 & 1 & 4 \\
\cline { 2 - 7 } & F3/F4 & 2 & 13 & 7 & 22 \\
\cline { 2 - 6 } & TOTAL & 5 & 25 & 12 & 42 \\
\hline
\end{tabular}

Tabela 15. Resultado da comparação do ARFI vs FIB4, segundo categoria Kappa.

\begin{tabular}{|l|l|l|l|l|}
\hline & F0/F1 & F2 & F3/F4 & Geral \\
\hline KAPPA DA CATEGORIA & 0.127 & 0.051 & 0.067 & 0.077 \\
\hline RESULTADOS & Concordância pobre & Concordância pobre & Concordância pobre & Concordância pobre \\
\hline
\end{tabular}

No estudo, observou-se que o nível de correlação dos métodos de elastografia, ARFI e FibroScan, com os métodos laboratoriais APRI e FIB-4, foi, respectivamente, Kappa 0.13 (tabela 2 e tabela 3) e kappa 0.107 (tabela 4 e tabela 5), mostrando pobre concordância. A análise comparativa dos métodos laboratoriais, APRI e FIB4, entre si, mostrou grau de concordância moderado com Kappa 0.663 (tabela 5 e tabela 6).

O FibroScan, quando comparado ao APRI, teve Kappa 0.138, demonstrando pobre concordância (tabela 8 e tabela 9). O grau de fibrose avançado $\mathrm{F} 3 / \mathrm{F} 4$, foi o que mostrou melhor concordância, porém, ainda assim com uma concordância pobre. Observou-se que o APRI tende a subestimar o grau de fibrose quando comparado ao FibroScan. Na correlação do FibroScan com o FIB-4 (tabela 10 e tabela 11) observou-se padrão semelhante, pobre concordância com Kappa (0.101), sendo melhor a concordância nos pacientes com fibrose avançada F3/F4. O FIB-4 tende a subestimar o grau de fibrose se comparado ao do FibroScan.
O ARFI, quando comparado ao APRI (tabela 12 e tabela 13), mostrou concordância pobre, Kappa 0.051, chamando atenção que nos estágios F2 e F3/F4, o valor de Kappa foi compatível com ausência de concordância. Na comparação do ARFI com o FIB4 (tabela 14 e tabela 15) o valor de Kappa foi de 0.077, também mostrando concordância pobre, tendo melhor correlação nos graus de fibrose inicial F0/F1. O FIB4 parece superestimar o grau de fibrose hepática quando comparado ao ARFI.

\section{DISCUSSÃO}

Embora percentualmente a incidência de HCC no Brasil não seja tão elevada como em outros países, assim mesmo, em valores absolutos, existem aqui cerca de 657.000 portadores virêmicos com necessidade de tratamento. Para este grupo de pacientes a determinação do grau de fibrose hepática é importante para a tomada de decisão sobre o tratamento medicamentoso e o acompanhamento após obter a resposta virológica sustentada, uma vez que aqueles com grau de fibrose 
F3 ou F4 necessitaram acompanhamento a cada 6 meses, tendo em vista o risco de carcinoma hepatocelular. Anteriormente, esta determinação era fator indispensável para indicar ou não o tratamento. Hoje, deixou de ser indispensável, mas ainda é importante para determinar a duração do tratamento e algumas variações dentre os medicamentos a serem utilizados. ${ }^{1,2}$

A determinação da fibrose através da biópsia hepática/exame histopatológico, apesar de ainda ser considerada o método padrão ouro, apresenta muitas desvantagens, tais como erro de amostragem na obtenção do parênquima hepático, discordância de análise mesmo entre patologistas experientes, baixa aceitação por parte do paciente, alto custo, possibilidade de muita dor no local da punção e, principalmente, riscos de sangramento, perfuração e infecção. Diante dessas desvantagens da biópsia hepática, justifica-se a valorização e a busca de consolidação dos métodos não invasivos.

Dentre as opções não invasivas de avaliação da fibrose hepática, os métodos de imagem (elastográficos) têm maior sensibilidade diagnóstica e são amparados por mais estudos do que os métodos sorológicos. Entretanto, exames como ARFI e FibroScan são mais caros e menos disponíveis, sobretudo em países subdesenvolvidos. A alta disponibilidade dos exames laboratoriais necessários ao cálculo do FIB4 e APRI é um fator atrativo para que se busque consolidar a utilidade desses métodos. No presente estudo, procuramos avaliar o grau de concordância do ARFI, FibroScan, APRI e FIB4 sem, no entanto, discutir quais deles possuíam melhor sensibilidade.

Partindo do grupo de pacientes acompanhados nos ambulatórios de HUWC e HSJ que já haviam sido submetidos a exames de elastografia, calculamos o grau de fibrose hepática pelo APRI e FIB4 e em seguida realizamos todas as possíveis combinações de correlações entre os quatro métodos (ARFI, FibroScan, APRI e FIB4).

Inicialmente, comparamos os resultados dos pacientes que realizaram ARFI e FibroScan, ou seja, um método de elastografia, com o cálculo do APRI (tabela 2 e 3) nesses pacientes. Estas comparações mostraram pobre concordância. Em seguida, comparamos os mesmos pacientes, com o cálculo do FIB4 (tabela 4 e 5), que também mostrou pobre concordância. Estes dados põem em questão a valorização do APRI e FIB4, pelo menos, como método isolado para determinação da fibrose hepática.

Posteriormente, comparamos os resultados dos exames sorológicos entre si (tabela 6 e 7), demonstrando concordância moderada a importante, dados que correspondem aos da literatura. $^{8}$

Seguindo, comparamos os resultados dos pacientes que possuíam FibroScan com o APRI (tabela 8 e 9), que igualmente, mostraram concordância pobre, fato coerente com os dados obtidos na soma dos resultados dos métodos de elastografia com o APRI.

Na avaliação dos resultados do FibroScan comparados com o
FIB4 (tabela 10 e 11) o grau de concordância foi igualmente pobre.

Observou-se, portanto, que os dois métodos sorológicos, quando comparados ao FibroScan, são discordantes na maioria das vezes.

Uma vez que o FibroScan é o método de elastografia mais consagrado e validado, e que existem estudos na literatura mostrando forte correlação deste com o método padrão ouro (biópsia hepática), pode-se considerar que os métodos sorológicos comparados no presente estudo têm baixa fidedignidade em avaliar o grau de fibrose hepática, visto que não mostraram forte de concordância com o FibroScan.

Outro método de elastografia disponível aos pacientes do nosso estudo foi o ARFI. O ARFI é mais recente, menos difundido e menos consagrado que o FibroScan. Porém, já existem estudos que o comparam aos outros métodos de elastografia e à biópsia hepática e o reputam como seguro e de boa sensibilidade para avaliação da fibrose hepática.

Em nosso estudo, comparamos isoladamente os pacientes que realizaram o ARFI com os métodos laboratoriais (APRI e FIB4) (tabela 12, 13, 14 e 15). Os resultados, tanto da comparação do ARFI com APRI, como do ARFI com FIB4, mostraram concordância pobre e até ausência de concordância (valor Kappa negativo) nos graus de fibrose mais acentuada (grupo F2/F3/F4) (tabela 12 e 13).

Estes dados nos levam, mais uma vez, a questionar a validade dos métodos laboratoriais, uma vez que, quando comparados aos métodos de elastografia, validados na literatura mundial, demonstram ausência de concordância ou apenas pobre concordância.

Devemos levar em consideração como fatores limitantes do presente estudo o número limitado de pacientes analisados (31 pacientes com FibroScan e 42 com ARFI), o fato de não terem sido excluídos pacientes com algumas comorbidades (coinfecção com vírus B ou Vírus da Imunodeficiência Humana, esteatose hepática, IMC elevado, níveis altos de Bilirrubina, AST, ALT), assim como, de alguns pacientes estarem em uso de medicamentos com possibilidade de alterar de algum modo os resultados. Outros fatores que podem ser considerados como limitantes se referem à realização dos exames de FibroScan por 3 examinadores diferentes e a realização dos exames laboratoriais em duas instituições diferentes e, por vezes, em momentos bastante distintos da realização das elastografias.

\section{CONCLUSÃO}

Pode-se concluir com base nos resultados obtidos que as comparações dos dois métodos laboratoriais (APRI e FIB4) com os dois métodos de elastografia validados (FibroScan e ARFI) têm predominantemente concordância pobre, com espectro de variação entre ausência de concordância e concordância moderada. 


\section{REFERÊNCIAS}

1. Ministério da Saúde. Comissão Nacional de Incorporação de Tecnologias no SUS (CONITEC). Protocolo clínico e diretrizes terapêuticas para Hepatite Ce coinfecções [Internet]. Brasília: Ministério da Saúde; 2018 [acesso en: 11 set 2018]. Disponível em: http://conitec. gov.br/images/Relatorios/2018/Relatorio_PCDT_HepatiteC.pdf

2. Ministério da Saúde. Comissão Nacional de Incorporação de Tecnologias no SUS [Internet]. Protocolo clínico e diretrizes terapêuticas para Hepatite $\mathrm{C}$ e coinfecções. Brasilia: Ministério da Saúde; 2015 [acesso em: 07 jul 2016]. Disponível em: http:// conitec.gov.br/images/Consultas/Relatorios/2015/Relatorio_ PCDTHepatiteC_CP.pdf.

3. Khairy M, Abdel-Rahman M, El-Raziky M, El-Akel W, Zayed N, Khatab H, et al. Non-invasive prediction of hepatic fibrosis in patients with chronic HCV based on the routine pre-treatment workup. Hepat Mon. 2012;12(11):e6718.

4. Stasi C, Milani S. Non-invasive assessment of liver fibrosis: between prediction/prevention of outcomes and cost-effectiveness. World J Gastroenterol. 2016;22(4):1711-20.

5. Arrayhani M, Sqalli T, Tazi N, El Youbi R, Chaouch S, Aqodad N, et al. Non-invasive assessment of liver fibrosis in chronic hemodialysis patients with viral hepatitis C. Pan Afr Med J. 2015;22:273.

6. Silva RG Junior, Schmillevitch J, Nascimento MF, Miranda
ML, Brant PE, Schulz PO, et al. Acoustic radiation force impulse elastography and serum fibrosis markers in chronic hepatitis C. Scand J Gastroenterol. 2014;49(8):986-92.

7. Li S, Li G, Fu D, Wang Y, Dang L. Liver fibrosis evaluation by ARFI and APRI in chronic hepatitis C. World J Gastroenterol. 2014;20(28):9528-33.

8. Cepeda JA, Solomon SS, Srikrishnan AK, Nandagopal P, Balakrishnan P, Kumar MS, et al. Serum fibrosis markers for the diagnosis of liver disease among people with chronic hepatitis $\mathrm{C}$ in Chennai, India Open Forum Infect Dis. 2016;3(3):ofw156.

9. Tachi Y, Hirai T, Toyoda H, Tada T, Hayashi K, Honda T, et al. Predictive ability of laboratory indices for liver fibrosis in patients with chronic Hepatitis $\mathrm{C}$ after the eradication of Hepatitis $\mathrm{C}$ virus. PLoS One. 2015;10(7):e0133515.

10. Gökcan H, Kuzu UB, Öztaş E, Saygıl1 F, Öztuna D, Suna $\mathrm{N}$. The predictive value of noninvasive serum markers of liver fibrosis in patients with chronic hepatitis C. Turk J Gastroenterol. 2016;27(2):156-64.

11. El-Sayed R, Fahmy M, El Koofy N, El-Raziky M, El-Hawary $\mathrm{M}$, Helmy $\mathrm{H}$, et al. Can aspartate aminotransferase to platelet ratio index replace liver biopsy in chronic hepatitis C? Trop Gastroenterol. 2011;32(4):267-72.

\section{Como citar:}

Mello FS, Lima JM, Hyppolito EB, Lima RV, Rolim FE, Pinho CS, et al. Comparação dos graus de fibrose hepática na hepatite C crônica (HCC) medidos por métodos de elastrografia e de sorologia: ARFI e FibroScan vs APRI e FIB4. Rev Med UFC. 2020 abr-jun;60(2):18-25. 